\title{
NOVA OCORRÊNCIA DE ASIATOCERATODUS (OSTEICHTHYES, DIPNOIFORMES) NA FORMAÇÃO ALCÂNTARA, EOCENOMANIANO DA BACIA DE SÃO LUÍS, MA, BRASIL
}

\author{
DARCILÉA FERREIRA CASTRO, CARLOS EDUARDO VIEIRA TOLEDO, \\ ELIANE PINHEIRODESOUSA \\ Pós-Graduação em Geociências, NEPV, IGCE, UNESP Campus de Rio Claro, 13506-900, SP, Brasil. \\ dfcastro@rc.unesp.br, cetoledo@rc.unesp.br,esousa@rc.unesp.br \\ MANUEL ALFREDO MEDEIROS \\ Departamento de Biologia, UFMA, Campus do Bacanga, 65080-040, São Luís, MA, Brasil. alf@elo.com.br
}

\begin{abstract}
RESUMO - O Cretáceo médio está bem representado na Bacia de São Luís pelos depósitos predominantemente areníticos da Formação Alcântara. Na Ilha do Cajual, norte do Estado do Maranhão, nordeste do Brasil, uma paleocomunidade continental datada como de idade eocenomaniana inclui vegetais, peixes, crocodilomorfos e dinossauros. Duas placas dentárias, coletadas em uma fácies conglomerática, com sete cristas arranjadas de forma progressivamente mais inclinada posteriormente, foram comparadas com padrões morfológicos de placas de dipnóicos do Cretáceo Superior do norte da África. A análise revelou que tal material é atribuível a Asiatoceratodus cf. tiguidiensis, uma espécie que ocorre ao longo da maior parte do Mesozóico e que previamente havia sido registrado no Albiano da África e do nordeste brasileiro, mas até o presente ainda não tinha sido confirmado no Cenomaniano brasileiro.
\end{abstract}

Palavras-chave: Asiatoceratodus, dipnóicos, Cretáceo, Formação Alcântara.

ABSTRACT - NEW OCCURRENCE OF DIPNOAN ASIATOCERATODUS IN THE ALCÂNTARA FORMATION, EARLY CENOMANIAN OF THE SÃO LUÍS BASIN, MARANHÃO STATE, BRAZIL. The middle Cretaceous is well represented in the predominantly arenitic deposits of Alcântara Formation. In the Cajual Island, Northern Maranhão state, Northeastern Brazil, a continental paleofauna dated as early Cenomanian includes plants, fishes, crocodiles and dinosaurs. Two small triangular dental plates, collected in a conglomeratic facies, presenting seven sharp ridges in a progressively posteriorly inclined arrangement, were compared with middle Cretaceous North African dipnoan plates. The analysis revealed that such material can be referred to Asiatoceratodus cf. tiguidensis a species that occurs through most of the Mesozoic and have already been recorded in the Albian of Africa and Northeastern Brazil, but was not confirmed in the Brazilian Cenomanian so far.

Key words: Asiatoceratodus, dipnoans, Cretaceous, Alcântara Formation

\section{INTRODUÇÃO}

A Bacia de São Luís é uma bacia costeira do tipo rifte, localizada ao norte do Estado do Maranhão. Nesta estão encerrados depósitos cretácicos que documentam um registro do Aptiano ao Eo-Cenomaniano e coincidem com a separação dos continentes sul-americano e africano (Mesner \& Wooldridge, 1964; Aranha et al. 1990). As rochas inseridas no conjunto sedimentar da Bacia de São Luís afloram em falésias litorâneas ao longo da Baía de São Marcos. Estes afloramentos incluem a Formação Alcântara, uma das sub-unidades do Grupo Itapecuru, com amplitude temporal do Neo-Albiano ao EoCenomaniano. Nesta unidade geológica predominam arenitos estratificados depositados em ambiente marinho raso, com níveis conglomeráticos intercalados (Pedrão et al. 1993; Rosseti \& Truckenbrodt, 1997 e Rosseti, 2001).
Um grande número de fósseis provenientes da Formação Alcântara, representados em sua maioria por vertebrados, entre estes peixes, crocodilomorfos e dinossauros, tem sido coletado e estudado (Medeiros, 2001; Medeiros \& Schultz, 2001, 2002). Descoberta em 1994, a Laje do Coringa reúne um variado conjunto de material fóssil retrabalhado de depósitos continentais (Corrêa Martins, 1995, 1996) documentando uma variedade de formas dinossaurianas como terópodos e saurópodos (Medeiros, 2001; Medeiros \& Schultz, 2001, 2002, 2003).

Entre os fósseis encontrados na Laje do Coringa existe um número considerável de placas dentárias de dipnóicos. Os dipnóicos surgiram no Eo-Devoniano (Schultze, 1993) e são encontrados até os dias atuais em três continentes: África, América do Sul e Austrália. Placas dentárias relacionadas a Ceratodus africanus Haug 1905 foram identificadas nos 
afloramentos da Baía de São Marcos (Cunha \& Ferreira, 1980; Dutra \& Malabarba, 2001; Medeiros, 2001; Medeiros \& Schultz, 2001).

No presente trabalho apresenta-se uma análise de material atribuído ao gênero Asiatoceratodus Vorob'yeva, 1967, coletado na Ilha do Cajual, Baía de São Marcos.

Segundo Toledo \& Bertini (2002), as poucas ocorrências de dipnóicos no Brasil se distribuem do Paleozóico ao Cenozóico, nas seguintes bacias: no Devoniano (Fernandes \& Carvalho, 2002), Permiano (Ragonha, 1989; Toledo, 2000, 2001; Toledo \& Bertini 1999, 2001, 2003; Richter \& Langer, 1998) e Triássico (Richter, 2001) da Bacia do Paraná (Estados do Rio Grande do Sul, Paraná, São Paulo e Mato Grosso); no Permiano da Bacia do Parnaíba (Silva Santos, 1989); Cretáceo da Bacia Bauru (Bertini et al., 1993); Juro-Cretáceo da Bacia do Araripe (Silva \& Azevedo, 1996); Cretáceo da Bacia de São Luís (Medeiros, 2001; Toledo \& Bertini, 2002; Medeiros \& Schultz, 2001, 2002; Sousa et al. 2003) e Cenozóico da Bacia do Acre (Silva Santos, 1987).

A raridade do grupo era um fator limitante ao estudo. Isto mudou nos últimos anos, com a descoberta de novas localidades, sendo que as mais importantes ocorrem nas bacias do Paraná (região de Rio Claro, Estado de São Paulo), São Luís (Estado do Maranhão) e Acre na região de Rio Branco (Toledo, 2001). Estas áreas apresentam maior abundância e variabilidade morfológica.

\section{MATERIALE MÉTODOS}

As duas placas dentárias aqui analisadas, UFMA 1.40.018 e UFMA 1.40.454, foram coletadas na Ilha do Cajual-MA (Figura 1), nos afloramentos Laje do Coringa e Falésia do Sismito, respectivamente distantes cerca de $200 \mathrm{~m}$ um do outro. As placas estavam dispersas entre os blocos do conglomerado fossilífero erodido pela ação das ondas do mar. No laboratório o material foi limpo e catalogado na Coleção Paleontológica da Universidade Federal do Maranhão (UFMA).

A análise morfológica, medidas tomadas e terminologia aqui aplicadas foram baseadas nas características propostas por Martin (1984). Foram utilizados como critérios diagnósticos, dimensões dos bordos, disposição e forma das cristas e disposição do ângulo-mesial.

Segundo Martin (1980) as diferenças entre as placas dentárias superiores e inferiores podem ser assim identificadas: (i) lado mesial convexo da primeira crista na placa dentária inferior, visível em vista dorsal; (ii) lado mesial da primeira crista nas placas superiores não visível na mesma vista; (iii) ângulo entre o plano da superfície oclusal da placa dentária, e o lado mesial da primeira crista, agudo nas placas superiores e obtuso nas inferiores; (iv) as faces labial e lingual das placas inferiores são retas ou levemente côncavas, e ocultas em vista posterior.

\section{SISTEMÁTICAPALEONTOLÓGICA}

\section{Ordem DIPNOI Müller, 1845 \\ Família ASIATOCERATODONTIDAE Vorob’yeva, 1967 Gênero ASIATOCERATODUS Vorob'yeva, 1967}

\section{Asiatoceratodus cf. tiguidiensis Tabaste, 1963}

Material. UFMA 1.40.454, placa dentaría completa; UFMA 1.40.018, placa dentaría incompleta.

Descrição. A placa dentária UFMA 1.40.454 (Figura 2), melhor preservada, é utilizada como padrão morfológico para a

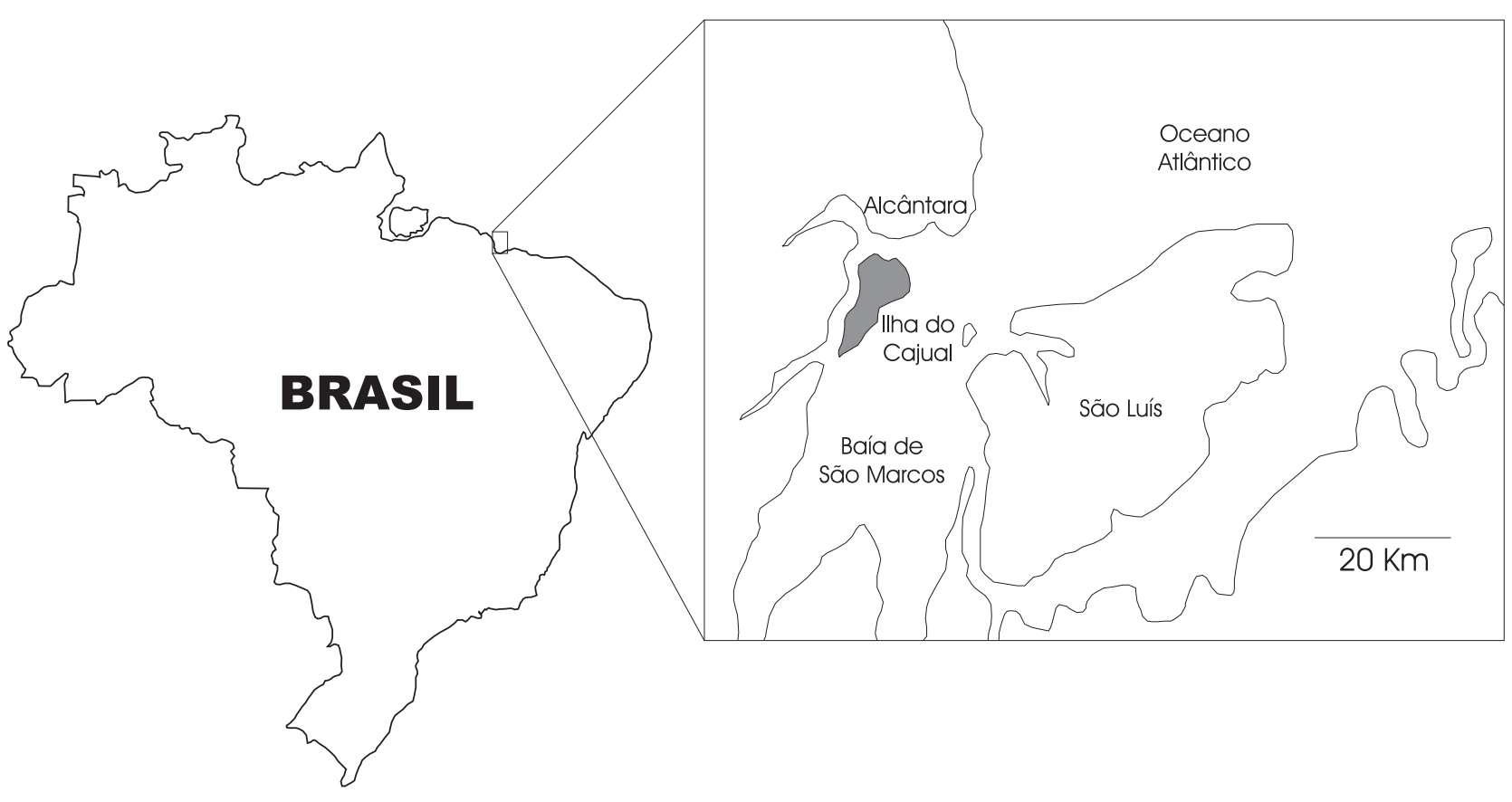

Figura 1. Localização da Laje do Coringa e Falésia do Sismito (Modificado de Carvalho, 2001).

Figure 1. Location of Laje do Coringa and Falésia do Sismito outcrops (modified from Carvalho, 2001). 


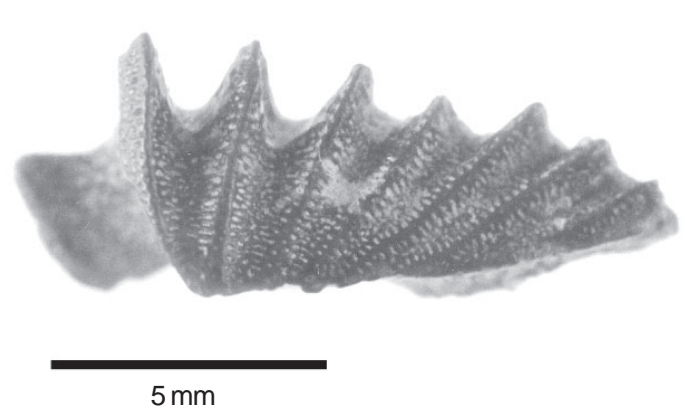

Figura 2. Asiatoceratodus sp., UFMA 1.40. 454, placa dentária (comprimento $=10,36 \mathrm{~mm}$ ).

Figure 2. Asiatoceratodus sp., UFMA 1.40. 454, dental plate (lenght $=10,36 \mathrm{~mm}$ ).

descrição. Trata-se de uma placa dentária inferior direita, completa e ainda preservando parte do pré-articular. Tem forma de triângulo retângulo, com 10,36mm de comprimento. Possui sete cristas cortantes retilíneas bem definidas, cuja orientação progressivamente inclina-se mais posteriormente, em ângulo mais fechado com a margem mesial, quanto mais posterior é a crista. O bordo mesial, com $4,81 \mathrm{~mm}$, demarcado pela primeira cúspide, curva-se levemente, delimitando um ângulo aberto. O bordo lingual, retilíneo, mede $9,74 \mathrm{~mm}$.

UFMA 1.40.018 é uma pequena placa superior direita, medindo 13,5 mm de comprimento. Esta placa apresenta sete cristas retilíneas, orientadas em direção posterior, porém está incompleta. As extremidades distais das três primeiras cúspides estão quebradas, assim como parte das duas últimas. A primeira crista é levemente côncava, voltada para extremidade distal da placa. O bordo lingual é retilíneo. $\mathrm{Na}$ superfície oclusal da placa observa-se uma suave elevação sobre o ângulo mesial (Figura 3).

Discussão. As características morfológicas dos espécimens UFMA 1.40 .454 e UFMA 1.40.018, preliminarmente identificado por Medeiros (2001) como Arganodus sp., permitem atribuí-los ao gênero Asiatoceratodus,

Com o estudo de UFMA 1.40.454, em melhor estado de preservação, é possível confirmar a presença de Asiatoceratodus na Formação Alcântara, em depósitos considerados de idade eocenomaniana (Pedrão et al., 1993).

Tabaste (1963) identificou várias placas de dipnóicos nos sedimentos cretáceos no Deserto do Saara, norte da África, descrevendo-as como Ceratodus tiguidiensis. Posteriormente o gênero Asiatoceratodus foi descrito, com base em crânio e placas dentárias, por Vorob’yeva (1967), para os depósitos triássicos da Rússia.

Placas dentárias similares às de Ceratodus tiguidiensis foram descritas por Martin (1979) em depósitos triássicos do Marrocos, criando o gênero Arganodus. Martin (1979) inclui em Arganodus a espécie descrita por Tabaste (1963), Arganodus tiguidensis. As placas dentárias referentes a Arganodus

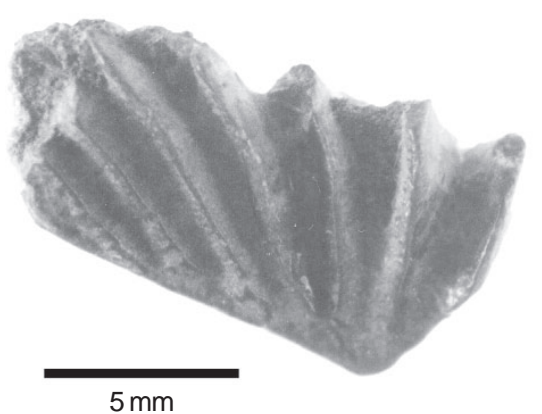

Figura 3. Asiatoceratodus sp., UFMA 1.40. 018, placa dentária (comprimento $=13,5 \mathrm{~mm}$ ).

Figure 3. Asiatoceratodus sp., UFMA 1.40. 018, dental plate (lenght $=13,5 \mathrm{~mm}$ )

apresentam geralmente forma de triângulo retângulo, sete cúspides retilíneas cortantes, que progressivamente inclinamse posteriormente, em ângulo mais fechado (Martin, 1979).

Kemp (1998), analisando ossos cranianos e placas dentárias, não encontrou diferenças significativas entre Asiatoceratodus e Arganodus, considerando-os como um mesmo gênero.

No norte do Estado do Maranhão a ocorrência de Asiatoceratodus foi registrada em depósitos do Albiano na região de Itapecuru Mirim (Bacia do Grajaú) por Dutra \& Malabarba (2001), com base em uma única placa superior direita. A ocorrência de Asiatoceratodus na Formação Alcântara, Cretáceo médio, amplia sua distribuição temporal para o Eocenomaniano.

$\mathrm{O}$ registro de Asiatoceratodus, juntamente com Ceratodus africanus, na Ilha do Cajual do Estado do Maranhão, vem reforçar a similaridade previamente observada por Medeiros (2001) e Medeiros \& Schultz $(2001,2002)$ entre a paleofauna da Ilha do Cajual e aquela do norte da África, registrada em depósitos cronocorrelatos, incluindo dipnóicos, outros grupos de peixes e dinossauros.

\section{AGRADECIMENTOS}

Agradecemos a Reinaldo J. Bertini (NEPV, UNESP Rio Claro) pela disponibilização de laboratórios e equipamentos utilizados para a análise do material. Aos revisores anônimos pelas críticas e sugestões à este manuscrito. Este trabalho teve suporte financeiro da UFMA, CPHNA-MA, CAPES e FAPESP(Processo 01/12575-0).

\section{REFERÊNCIAS}

Aranha, L.G. de; Lima, H.P; Souza, J.M.P. de \& Makino, R.K. 1990. Origem e evolução das bacias de Bragança-Viseu, São Luís e Ilha Nova. In: G.P.R. Gabaglia \& E.J. Milani (eds.) Origem e Evolução de Bacias Sedimentares, Petrobrás, p. 221-233.

Bertini, R.J.; Marshall, L.G.; Gayet, M. \& Brito, P.1993. Vertebrate faunas from the Adamantina and Marília formations (Upper Baurú Group, Late Cretaceous, Brazil) in their stratigraphic and paleobiogeographic context. Neues Jabrbuch für Geologie und Palaontologie (Abhandlungen), 188(1): 71-101. 
Carvalho, I.S. 2001. Pegadas de dinossauros em depósitos estuarinos (Cenomaniano) da Bacia de São Luís (MA), Brasil. In: D.F. Rosseti; A.M. Góes \& W. Truckenbrodt (eds.) $O$ Cretáceo na Bacia de São Luís-Grajaú, MPEG, p. 245-264.

Corrêa Martins, F. J. 1995. Sensoriamento Remoto: Perspectivas e aplicações. Análise de caso: Bacia de São Luís, MA: In: REUNIÃO ANUAL DA SBPC, 47, 1995. Resumos, Belém, SBPC, p. 433.

Corrêa Martins, F.J. 1996. Levantamento de aspectos geológicos da parte setentrional da ilha de São Luís e áreas adjacentes. Programa de Pós-Graduação em Geociências, Universidade Federal do Rio de Janeiro, Dissertação de Mestrado, 98 p.

Cunha, F.L. \& Ferreira, C.S. 1980. Um dipnoi na Formação Itapecuru (Cenomaniano), Maranhão, Brasil: In: CONGRESSO LATINOAMERICANO DE PALEONTOLOGIA Y BIOESTRATIGRAFIA Y CONGRESSO LATINOAMERICANO DE PALEONTOLOGIA, 2/1, 1980. Actas, Buenos Aires, APA, p.1-9.

Dutra, M.F.A. \& Malabarba, M.C.S.L. 2001. Peixes do AlbianoCenomaniano do Grupo Itapecuru no estado do Maranhão, Brasil. In: D.F. Rossetti; A.M. Góes \& W. Truckenbrodt (eds.) o Cretáceo da Bacia de São Luís-Grajaú, MPEG, p. 191-208.

Fernandes, A.S. \& Carvalho, I.S. 2002. Uma provável escavação de Dipnóico na Formação Ponta Grossa, Devoniano da Bacia do Paraná. Arquivos do Museu Naional, 60(3):207-211.

Haug, E. 1905. Paléontologie. In: F. Foureau (ed.) Documents scientifiques de la mission saharienne. Mission Foureau-Lamy "D'Alger au Congo par le Tchad 1904-1905", Masson \& Cie, p. 751-832.

Kemp, A. 1998. Skull structure in post-paleozoic lungfish. Journal of Vertebrate Paleontology, 18(1): 43-63.

Martin, M. 1979. Arganodus atlantis et Ceratodus arganensis, deux nouveaux dipneustes du Trias supérieur continental marocain. Compte Rendu de l'Academie de Sciences Paris, 289: 89-92.

Martin, M. 1980. Revision of Ceratodus concinnus Plieninger. Stuttgarter Beitr. Naturkde, Series B, 56:1-15.

Martin, M. 1984. Révision des Arganodontidés et des Néocératodontidés (Dipnoi, Ceratodontiformes) du Crétacé africain. Neues Jahrbuch für Geologie und Paläontologie (Abhandlungen), 169(2): 225-260.

Medeiros, M.A. 2001. A Laje do Coringa (Ilha do Cajual, Bacia de São Luís, baía de São Marcos, MA): conteúdo fossilífero, bioestratinomia, diagênese e implicações na paleobiogeografia do Mesocretáceo do nordeste brasileiro. Programa de Pós-Graduação em Geociências, Universidade Federal do Rio Grande do Sul, Tese de Doutorado, 137 p.

Medeiros, M.A. \& Schultz, C.L. 2001. Uma paleocomunidade de vertebrados do Cretáceo Médio, Bacia de São Luís. In: D.F. Rossetti; A.M. Góes, \& W. Truckenbrodt (eds.) O Cretáceo na Bacia de São Luís - Grajaú, MPGE, p. 209-221.

Medeiros, M.A. \& Schultz, C.L. 2002. A fauna dinossauriana da Laje do Coringa, Cretáceo médio do Nordeste do Brasil. Arquivos do Museu Nacional, 60(3): 155-162.

Medeiros, M.A. \& Schultz, C.L. 2003. Rebbachisaurus (Sauropoda) na Formação Alcântara, Cenomaniano do Maranhão. In : CONGRESSO BRASILEIRO DE PALEONTOLOGIA, 18, 2003. Resumos, Brasília, SBP, p. 187-188.

Mesner, J.C. \& Wooldridge, P. 1964. Estratigrafia das bacias paleozóicas e cretáceas do Maranhão. Petrobrás, p. 137-164 (Boletim 7).

Pedrão, E; Arai, M; Carvalho, I.S. \& Santos, M.H.B. 1993. Palinomorfos da Formação Itapecurú - análise palinológica de uma amostra de superfície da Ponta do Farol, São Luís MA. Petrobrás, Cenpes, 10 p. (Relatório técnico).

Ragonha, E.W. 1989. Placas dentárias de Dipnoi no Grupo Passa Dois (P-Tr) da Bacia do Paraná. Apreciações ambientais, climáticas, cronológicas e estratigráficas: In: CONGRESSO BRASILEIRO PALEONTOLOGIA, 11, 1989. Anais, Curitiba, SBP, 1: 195-206.
Richter, M. 2001. First lungfish (Sarcopterygii:Dipnoi) from the Santa Maria Formation, Triassic of Rio Grande do Sul State, Brazil. In: CONGRESSO BRASILEIRO DE PALEONTOLOGIA, 17, 2001. Resumos, Rio Branco, SBP, p.121.

Richter, M. \& Langer, M.C. 1998. Fish remains from the Upper Permian Rio do Rasto Formation (Paraná Basin) of Southern Brazil. Journal of African Earth Sciences, 27: 158-159.

Rossetti, D.F. 2001. Arquitetura deposicional da Bacia de São LuísGrajaú. In: D.F Rossetti; A.M. Góes \& W. Truckenbrodt (eds.) O Cretáceo da Bacia de São Luís-Grajaú, MPEG, p. 31-46.

Rossetti, D.F. \& Truckenbrodt, W. 1997. Revisão estratigráfica para os depósitos do Albiano - Terciário Inferior (?) na bacia de São Luís (MA), norte do Brasil. Boletim do Museu Paraense Emílio Goeldi (Série Ciências da Terra), 9: 29-41.

Schultze, H. - P. 1993. Osteichthyes: Sarcopterygii. In: The Fossil Record 2 (M. J. Benton, ed.) London: 657-663. Chapman \& Hall.

Silva Santos, R. 1987. Lepidosiren megalos n. sp. do Terciário do Estado do Acre-Brasil. Anais da Academia Brasileira de Ciências, 59: 375-384.

Silva Santos, R. 1989. A ictiofaúnula da Formação Pedra de Fogo, Bacia do Parnaíba, NE do Brasil: Sarcopterygii-Actinista e Dipnoi. In: CONGRESSO BRASILEIRO DE PALEONTOLOGIA, 11, 1989. Resumos, Curitiba, SBP, p. 33-34.

Silva, V.G. \& Azevedo, S.A. 1996. Um dipnóico da Formação Brejo Santo, Jurássico da Chapada do Araripe, Ceará, Brasil. Anais da Academia Brasileira de Ciências, 64: 419-420.

Sousa, E.P.; Toledo, C.E.V. \& Medeiros, M.A. 2003. Novas ocorrências de dipnóicos no Cretáceo Médio da Ilha do Cajual, Estado do Maranhão: In: CONGRESSO BRASILEIRO DE PALEONTOLOGIA, 18, 2003. Resumos, Brasília, SBP, p. 282 $-283$.

Tabaste, N. 1963. Étude de restes de poissons du crétacé saharien. Mélanges ichthyologiques, Mémoire IFAN , 68: 437-485.

Toledo, C.E.V. 2000. Fossil Fishes from Corumbataí Formation, Paraná Basin, São Paulo, Brazil. In: ANNUAL MEETING OF THE SOCIETY OF VERTEBRATE PALEONTOLOGY, 60, 2000. Abstracts, Lawrence, SVP, p. 73.

Toledo, C.E.V. 2001. Análise paleoicitológica da Formação Corumbataí na região de Rio Claro, Estado de São Paulo. Programa de Pós-graduação em Geociências, Universidade Estadual Paulista, Dissertação de Mestrado, 146 p.

Toledo, C.E.V. \& Bertini, R.J. 1999. A paleoictiofauna da Formação Corumbataí no Estado de São Paulo: In: CONGRESSO BRASILEIRO PALEONTOLOGIA, 16, 1999. Resumos, Crato, SBP, p. 119-120.

Toledo, C.E.V. \& Bertini, R.J. 2001. Uma nova ocorrência de Dipnoicos e Petalodontes na Formação Corumbataí (Permiano Superior da Bacia do Paraná) na região de Angatuba, Estado de São Paulo. In: CONGRESSO BRASILEIRO DE PALEONTOLOGIA, 17, 2001. Resumos, Rio Branco, SBP, p. 128.

Toledo, C.E.V. \& Bertini, R.J. 2002. Occurrences of fossil dipnoi in Brazil and their stratigraphic and chronologic distributions. In: INTERNATIONAL PALAEONTOLOGICAL CONGRESS, 1, 2002. Abstracts, Sidney, IPA, 3: 284 - 285.

Toledo, C.E.V. \& Bertini, R.J. 2003. Stratigraphy and vertebrate paleontology of the Corumbatai Formation, Upper Permian from the Paraná Basin in the State of São Paulo, southeastern Brasil. In: INTERNATIONAL CONGRESS ON CARBONIFEROUS AND PERMIAN STRATIGRAPHY, 15, 2003. Abstracts, Utrecht, p.539-540.

Vorob'yeva, E.L. 1967. Triassic ceratod from south Fergana and remarks on the systematics and phylogeny of ceratodontids. PaleontologyJournal, 4: 102-111. 